\title{
Joint Registration and Limited-Angle Reconstruction of Digital Breast Tomosynthesis
}

\author{
Guang Yang ${ }^{\dagger}$, John H. Hipwell ${ }^{1}$, Christine Tanner ${ }^{2}$, David J. Hawkes ${ }^{1}$, and \\ Simon R. Arridge ${ }^{1}$ \\ ${ }^{1}$ Centre for Medical Image Computing, Department of Computer Science and \\ Medical Physics, University College London (UCL), London, WC1E 6BT, UK \\ ${ }^{2}$ Computer Vision Lab, Eidgenössische Technische Hochschule, Zürich, 8092, CH
}

\begin{abstract}
Digital breast tomosynthesis (DBT), an emerging imaging modality, provides a pseudo-3D image of the breast. Algorithms to aid the human observer process these large datasets involve two key tasks: reconstruction and registration. Previous studies separated these steps, solving each task independently. This can be effective if reconstructing using a complete set of data, e.g., in cone beam CT, assuming that only simple deformations exist. However, for ill-posed limited-angle problems such as DBT, estimating the deformation is complicated by the significant artefacts associated with DBT reconstructions, leading to severe inaccuracies in the registration. In this paper, we present an innovative algorithm, which combines reconstruction of a pair of temporal DBT acquisitions with their simultaneous registration. Using various computational phantoms and in vivo DBT simulations, we show that, compared to the conventional sequential method, jointly estimating image intensities and transformation parameters gives superior results with respect to reconstruction fidelity and registration accuracy.
\end{abstract}

\section{Introduction}

Digital breast tomosynthesis (DBT) involves acquiring a small number of low dose X-ray images, over a limited angle, and reconstructing this data into a pseudo-3D image of the breast [1]. It is of considerable interest to the research community [2], as a potential replacement for conventional mammography, but has been slow to be adopted into routine clinical practice.

In a breast cancer screening or diagnostic setting, radiologists routinely compare conventional current and prior mammograms to detect suspicious changes that might be indicative of malignancy. DBT has the potential to improve the sensitivity and/or specificity of this task by reducing the confounding influence of overlaying tissue, but only if the large quantity of data acquired can be efficiently incorporated into the clinical workflow [3] [4] [5]. To enable the data to be viewed as a pseudo-3D volume, it must first be reconstructed. Although

\footnotetext{
${ }^{\dagger}$ Contact Email: G.Yang@cs.ucl.ac.uk. This work has been funded by DTI Project Digital Breast Tomosynthesis TP/7/SEN/6/1/M1577G. The authors would like to thank the UK MR Breast Screening Study (MARIBS) [6] for providing the data for this study.
} 
not currently a component of routine clinical practice, image registration algorithms could be used to aid the clinician in comparing temporal data sets. This would enable image features to be transformed into a common coordinate system where abnormal differences due to disease progression can be distinguished from differences due to patient position and breast deformation.

In other modalities, such as MRI or CT, registration has generally been performed after the images have been reconstructed. In DBT however, the presence of reconstruction artefacts due to the not insignificant null space, complicates the registration process. Rather than separate these two tasks and perform them sequentially therefore, we investigate an algorithm which performs them simultaneously, and test the hypothesis that the performance of the joint estimation will benefit both processes.

In the following sections we describe this algorithm and present a comparison of its performance with the sequential alternative. We test and validate the methods using phantom data and DBT simulations generated from breast MRI. Breast MRI is a fully 3D imaging modality which provides good visibility of internal breast anatomy. It is therefore a good surrogate source of breast data with which to test the performance of our algorithms.

\section{Method}

Two sets of limited angle X-ray acquisitions, $\boldsymbol{y}_{\mathbf{1}} \in \mathbb{R}^{N_{2}}$ and $\boldsymbol{y}_{\mathbf{2}} \in \mathbb{R}^{N_{2}}$, obtained at different times, can be expressed in terms of a $3 \mathrm{D}$ volume, $\boldsymbol{x} \in \mathbb{R}^{N_{3}}$, in two positions related by the transformation, $R$, with parameters, $\zeta_{p} \in \mathbb{R}^{n}$, and the system matrix $A: \mathbb{R}^{N_{3}} \mapsto \mathbb{R}^{N_{2}}$ (where $N_{2}$ is the projection dimension and $N_{3}$ is the volume dimension) via

$$
\boldsymbol{y}_{1}=A \boldsymbol{x}
$$

and

$$
\boldsymbol{y}_{\mathbf{2}}=A \boldsymbol{x}^{\dagger}=A R_{\zeta_{p}} \boldsymbol{x}
$$

Rather than perform the two tasks sequentially or iteratively $[7,8]$, we propose a fully coupled algorithm using a simultaneous reconstruction and registration framework summarised in Algorithm 1.

The objective function is given by

$$
\min _{\boldsymbol{x}, \boldsymbol{\zeta}_{p} \in \mathbb{R}^{n}} \Phi_{\mathrm{RR}}=\frac{1}{2}\left(\left\|A \boldsymbol{x}-\boldsymbol{y}_{\mathbf{1}}\right\|_{2}^{2}+\left\|A R_{\zeta_{p}} \boldsymbol{x}-\boldsymbol{y}_{\mathbf{2}}\right\|_{2}^{2}\right) .
$$

We combine optimisation of the two temporal reconstructions with the 12 degrees of freedom $\boldsymbol{\zeta}_{\boldsymbol{p}},(p=1,2, \ldots, 12)$, of an affine transformation, which globally describes the translation, scaling, rotation and shearing between the two time points. We can also substitute other non-rigid deformations for the affine transformation in this framework, but considered an affine transformation in the first instance due to its simplicity. 
In addition, we can also derive the gradient with respect to the image intensities $\boldsymbol{x}$ and transformation parameters $\zeta_{p}$ as follows:

$$
\begin{aligned}
\Psi_{\boldsymbol{x}} & =A^{T}\left(A \boldsymbol{x}-\boldsymbol{y}_{\mathbf{1}}\right)+R_{\boldsymbol{\zeta}_{p}}^{T} A^{T}\left(A R_{\boldsymbol{\zeta}_{p}} \boldsymbol{x}-\boldsymbol{y}_{\mathbf{2}}\right), \\
\Psi_{\boldsymbol{\zeta}_{p}} & =\left(A R_{\boldsymbol{\zeta}_{p}}^{\prime} \boldsymbol{x}\right)^{T}\left(A R_{\boldsymbol{\zeta}_{p}} \boldsymbol{x}-\boldsymbol{y}_{\mathbf{2}}\right) .
\end{aligned}
$$

To minimise issues of memory usage associated with processing these large datasets, we opt for a Quasi-Newton (L-BFGS) solver.

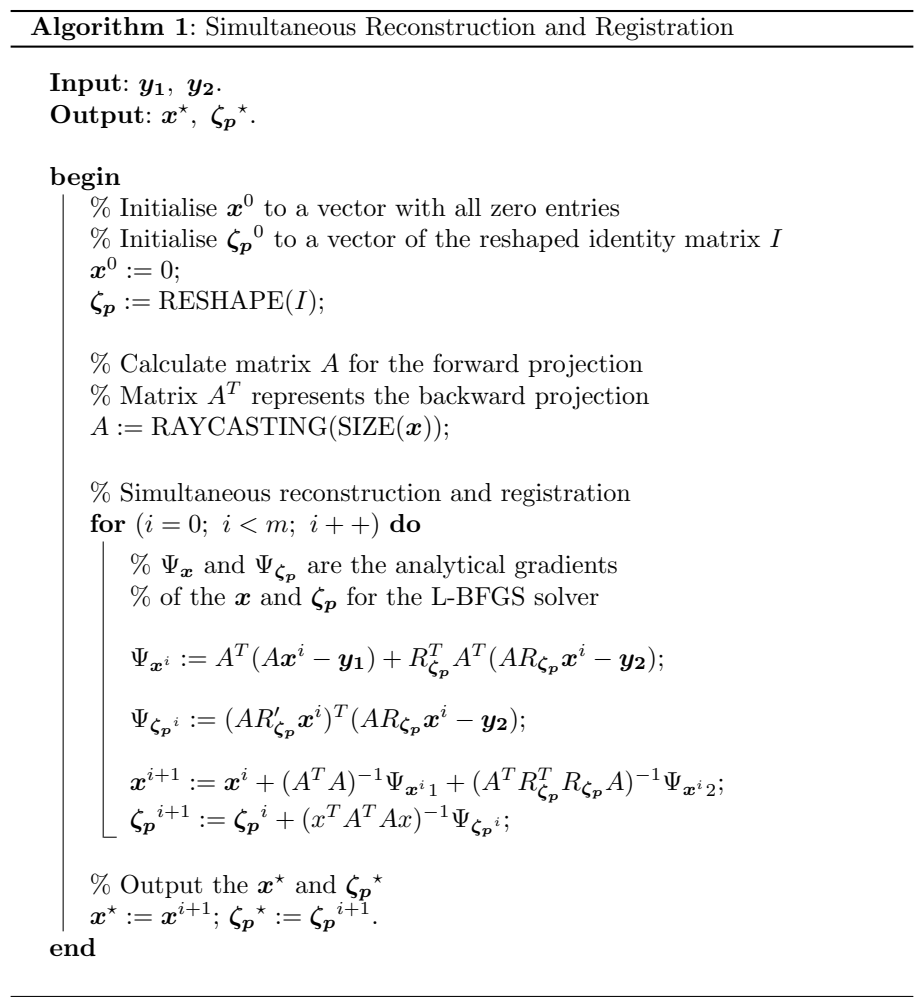

\section{Results}

In the following three experiments we compare the performance of (a) a sequential reconstruction and registration, in which $n=1000$ iterations of the reconstruction of projection images, $\boldsymbol{y}_{\mathbf{1}}$ and $\boldsymbol{y}_{\mathbf{2}}$, is followed by a single registration of the reconstructed volumes $\boldsymbol{x}_{\mathbf{1}}$ and $\boldsymbol{x}_{\mathbf{2}}(m=1)$ and (b) our simultaneous approach in which $n=50$ iterations of the reconstruction are followed by a registration and the process repeated $m=20$ times. In both cases the total number of iterations is the same $(m \times n=1000)$. Our test data is created from a 3D data set, $\boldsymbol{x}$, which is transformed by a known transformation to produce a second volume $\boldsymbol{x}^{\dagger}$. From each of these, 11 projections covering \pm 25 degrees are created 
to simulate the pair of temporal DBT acquisitions $\boldsymbol{y}_{\mathbf{1}}$ and $\boldsymbol{y}_{\mathbf{2}}$. In all experiments the affine transformation parameters were selected from random uniform distributions with the following limits: \pm 20 degrees for rotation, \pm 5 pixels for translation, 0.9 to 1.1 for the scale factor and a small amount of shearing.

In the first experiment, a 3D toroidal phantom image was created, and subjected to 20 affine transformations to test the robustness of our simultaneous method. The simultaneous results are much more compact and accurate than the sequential results, and the out of plane blurring is reduced (Fig. 1 (d)-(f) vs. Fig. $1(\mathrm{~m})-(\mathrm{o}))$. In the second experiment, 15 randomly generated affine transformations were applied to a 3D breast MR image and similar performance was observed (Fig. 2 (d)-(f) vs. Fig. 2 (m)-(o)). The specific parameters recovered are shown in Figs. 3 and 4. In a third experiment, we tested the methods using two MRI acquisitions obtained before and after application of a lateral-to-medial plate compression of the breast. There is no ground truth for the deformation of this dataset, however from both the image appearance (Fig. 5 (d)-(f) vs. Fig. $5(\mathrm{~m})-(\mathrm{o}))$ and the mean squared error (MSE in Table 1), we can conclude that our simultaneous method outperformed the sequential method.

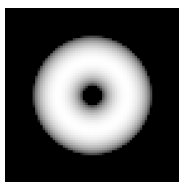

(a)

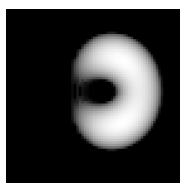

(d)

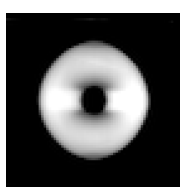

(g)

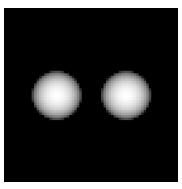

(b)

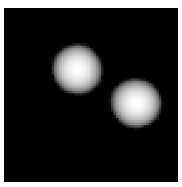

(e)

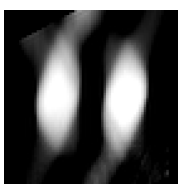

(h)

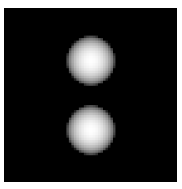

(c)

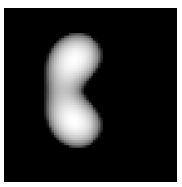

(f)

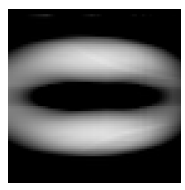

(i)

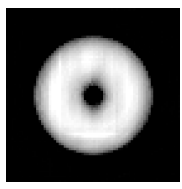

(j)

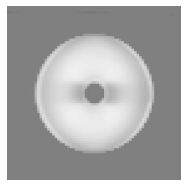

(m)

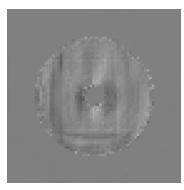

(p)

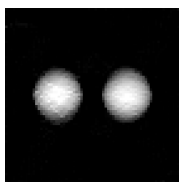

(k)

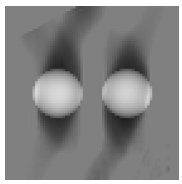

(n)

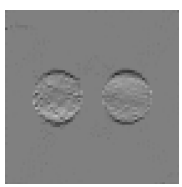

(q)

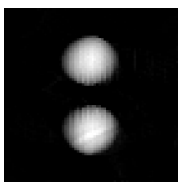

(1)

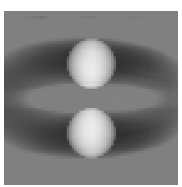

(o)

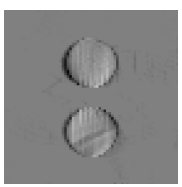

(r)

Fig. 1. Test case 1: Toroid phantom image. (a)-(c): Fixed image; (d)-(f): Moving image; (g)-(i): Sequential result, i.e., transformed moving image reconstruction; (j)-(l): Simultaneous result; $(m)-(o)$ : Difference between the sequential result and the fixed image; $(p)-(r)$ : Difference between the simultaneous result and the fixed image. (For each set of three sub-figures: Left: Coronal view; Middle: Transverse view; Right: Sagittal view.) 


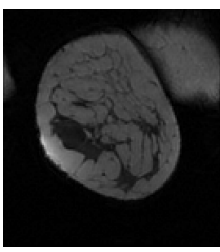

(a)

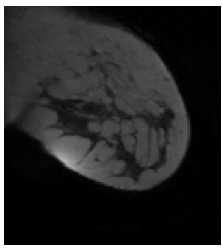

(d)

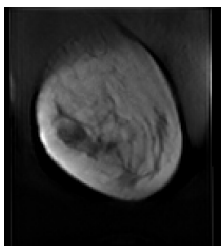

(g)

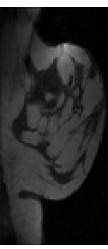

(b)

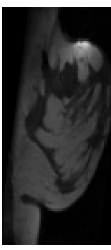

(e)

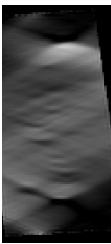

(h)

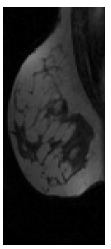

(c)

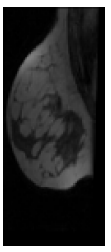

(f)

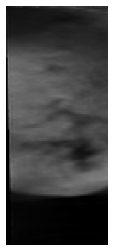

(i)

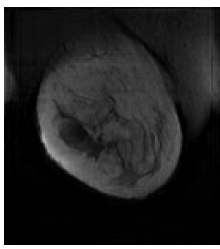

(j)

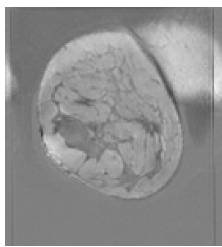

(m)

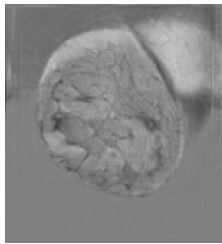

(p)

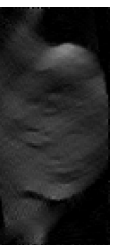

(k)

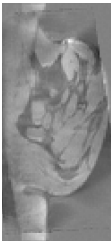

(n)

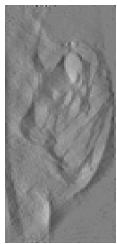

(q)

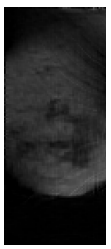

(l)

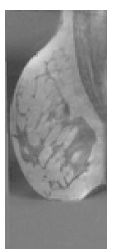

(o)

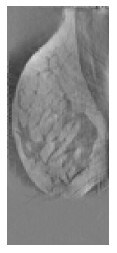

(r)

Fig. 2. Test case 2: $3 D$ breast $M R$ image. (a)-(c): Fixed image; (d)-(f): Moving image; (g)-(i): Sequential result, i.e., transformed moving image reconstruction; (j)-(l): Simultaneous result; $(m)$-(o): Difference between the sequential result and the fixed image; $(p)-(r)$ : Difference between the simultaneous result and the fixed image. (For each set of three sub-figures: Left: Coronal view; Middle: Transverse view; Right: Sagittal view.)

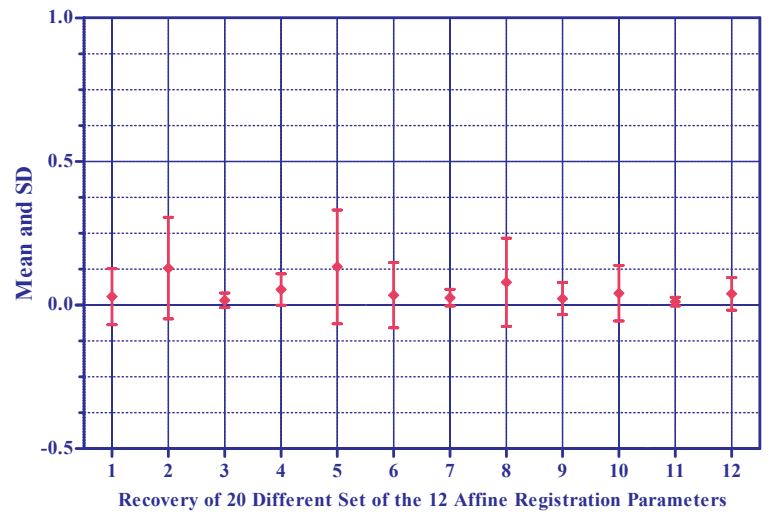

Fig. 3. The first experiment on a $3 D$ toroidal phantom image. The Mean and standard deviation of the absolute error between the recovered and the ground truth of 20 different sets of affine transformations. Parameters 4, 8, and 12 are the translations along each axis. 


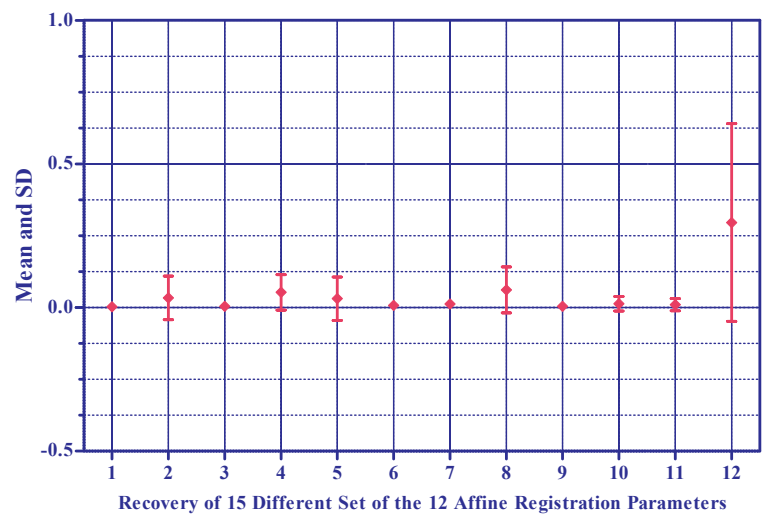

Fig. 4. The second experiment on $3 D$ breast MRI. The Mean and standard deviation of the absolute error between the recovered and the ground truth of 15 different sets of affine transformations. Parameters 4, 8, and 12 are the translations along each axis.

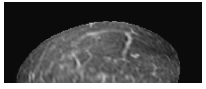

(a)

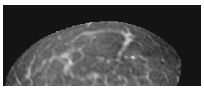

(d)

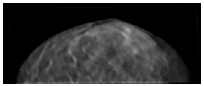

(g)

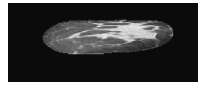

(b)

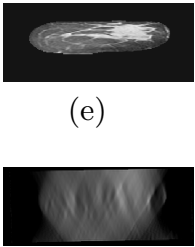

(h)

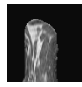

(c)

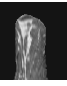

(f)

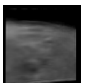

(i)

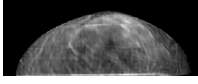

$(\mathrm{j})$

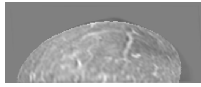

(m)

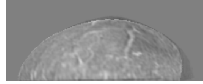

(p)

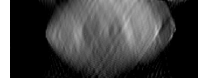

$(\mathrm{k})$

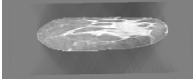

(n)

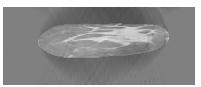

(q) (o)

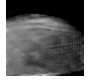

(1)
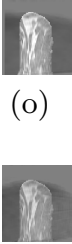

(r)

Fig. 5. Test case 3: DBT simulation with in vivo compression. (a)-(c): Fixed image; (d)-(f): Moving image; ( $g$ )-(i): Sequential result, i.e., transformed moving image reconstruction; (j)-(l): Simultaneous result; $(m)-(o)$ : Difference between the sequential result and the fixed image; $(p)-(r)$ : Difference between the simultaneous result and the fixed image. (For each set of three sub-figures: Left: Coronal view; Middle: Transverse view; Right: Sagittal view.)

Table 1. Comparison of the MSE error $\frac{1}{N_{3}}\left\|\boldsymbol{x}^{\star}-\boldsymbol{x}\right\|_{2}^{2}\left(N_{3}\right.$ is the number of voxels $)$.

\begin{tabular}{llll}
\hline & Initial & Sequential Method & Simultaneous Method \\
\hline Toroid Phantom & $1.31 \times 10^{6}$ & $7.46 \times 10^{3}$ & $0.24 \times 10^{3}$ \\
\hline Uncompressed Breast MRI & $1.18 \times 10^{6}$ & $6.04 \times 10^{3}$ & $3.01 \times 10^{3}$ \\
\hline In vivo DBT simulation & $5.32 \times 10^{6}$ & $3.68 \times 10^{4}$ & $3.22 \times 10^{4}$ \\
\hline
\end{tabular}




\section{Discussion}

As far as we aware this is the first time that the simultaneous reconstruction and registration of DBT data sets using a unified optimisation framework has been demonstrated to be superior to the conventional sequential method. This approach jointly considers reconstruction and registration components of DBT, and it is capable of recovering both the deformation parameters, and an enhanced, reconstructed image. By integrating the registration directly into the framework of the reconstruction problem, we are able to fully explore the interdependence between the transformation parameters and the $3 \mathrm{D}$ volume to be reconstructed.

Significantly, compared to previous research on combining reconstruction and registration (or motion correction), our combined limited angle DBT problem has a much larger null space and is severely ill-posed, which makes the inverse problem more intriguing and more challenging. From Table 2, we can see that for a typical 2D super-resolution problem previous studies used 5 low resolution images to restore a high resolution image recovering only rotations and translations, and 32 low resolution images for the affine registration. In general 3D problems, the authors used at least 60 and up to 799 forward projections covering a full-range of views, i.e., 180 degrees or 360 degrees, to perform the joint estimations. However, for our DBT application, we have two sets of data which are observed at two time-points. Each of the data is acquired using only 11 forward projections covering just 50 degrees ( \pm 25 degrees), and the two data sets overlap to a certain degree according to the original unknown deformations.

Table 2. Comparison of different applications of simultaneous inverse problem. (SR: super-resolution; LR: low resolution; fwdProjs: forward projections; Recon.+Regn.: reconstruction and registration; "-": not mentioned; Data collected according to [9]).

\begin{tabular}{lcccc}
\hline Publications & Application & Dimension & Optimiser & Data \\
\hline Chung et al. 2006 & SR & 2D Affine & Gauss-Newton & 32 LR images \\
\hline He et al. 2007 & SR & 2D Rigid & Conjugate Gradient & 5 LR images \\
\hline Yap et al. 2009 & SR & 2D Rigid & Linear Interior Point & 5 LR images \\
\hline Jacobson and Fessler 2003 & PET & 3D Affine & Gradient Descent & 64 fwdProjs $180^{\circ}$ \\
\hline Fessler 2010 & PET & 3D - & Conjugate Gradient & - \\
\hline Odille et al. 2008 & MRI & 3D Affine & GMRES & - \\
\hline Schumacher et al. 2009 & SPECT & 3D Rigid & Gauss-Newton & 60 to 64 fwdProjs $360^{\circ}$ \\
\hline Yang et al. 2005 & Cryo-EM & 3D Rotation & Quasi-Newton (L-BFGS) & 84 fwdProjs \\
\hline Chung et al. 2010 & Cryo-EM & 3D Rigid & Quasi-Newton (L-BFGS) & 799 fwdProjs \\
\hline Our Recon.+Regn. Model & DBT & 3D Affine & Conjugate Gradient or L-BFGS & 22 fwdProjs 50 $50^{\circ}\left( \pm 25^{\circ}\right)$ \\
\hline
\end{tabular}

We analysed our simultaneous method with various data sets using an affine transformation model, and the simultaneous method has clearly achieved superior results compared to the conventional sequential method. First, the experiment on the 3D toroid image demonstrates the potential of this approach over the conventional method to increase the depth resolution of the recontructed image. Second, the results of the breast MR image have further strengthened our 
confidence in the hypothesis that the reconstruction and registration have a reciprocal relationship. In addition, the recovery of the transformation parameters was consistently accurate for both the 3D toroid and the breast MR data sets. Next, we attempted to reconstruct and register simulated DBT data sets created from real medio-lateral compressions of a breast imaged using MRI. As anticipated, the simultaneous approach still outperformed the conventional sequential method as demonstrated by the image appearance and MSE comparison (Figure 5 and Table 1). Although the improvements were modest in this experiment, this can be attributed, at least in part, to the fact that the affine transformation, which is a global parametric model, is insufficient to capture such a non-rigid breast deformation.

\section{Conclusion}

We have presented a method to simultaneously reconstruct and register temporal DBT datasets and compared it with performing the two tasks sequentially. Our simultaneous method produced superior results in both registration accuracy and reconstructed image appearance. In future work we will incorporate B-spline transformations and address the application to combine reconstruction and registration of two view (cranial-caudal/mediolateral-oblique) DBT data sets, to overcome the null-space limitation.

\section{References}

[1] Niklason, L.T., et al.: Digital Tomosynthesis in Breast Imaging. Radiology, Vol. 205(2), 399-406 (1997)

[2] Dobbins III, J. T., et al.: Digital X-ray Tomosynthesis: Current State of the Art and Clinical Potential. Physics in Medicine and Biology, Vol. 48(19), p. R65 (2003)

[3] Poplack, S. P., et al.: Digital Breast Tomosynthesis: Initial Experience in 98 Women with Abnormal Digital Screening Mammography. American Journal of Roentgenology, Vol. 189(3), pp. 616-623 (2007)

[4] Gur, D., et al.: Digital Breast Tomosynthesis: Observer Performance Study. American Journal of Roentgenology, Vol. 193(2), pp. 586-591 (2009)

[5] Spangler, M. L., et al.: Detection and Classification of Calcifications on Digital Breast Tomosynthesis and 2D Digital Mammography: A Comparison. American Journal of Roentgenology, Vol. 196, pp. 320-324 (2011)

[6] Leach M.O., et al.: Screening with MRI and mammography of a UK population at high familial risk of breast cancer. The Lancet 365, pp. 1769-1778 (2005)

[7] Yang, G., et al.: Combined reconstruction and registration of digital breast tomosynthesis. Digital Mammography (IWDM'10), Lecture Notes in Computer Science, Vol. 6136, pp. 760-768 (2010)

[8] Yang, G., et al.: Combined reconstruction and registration of digital breast tomosynthesis: Sequential method versus iterative method. Medical Image Understanding and Analysis (MIUA'10), University of Warwick, Coventry, pp. 1-5 (2010)

[9] Yang, G.: Numerical Approaches for Solving the Combined Reconstruction and Registration of Digital Breast Tomosynthesis, Ph.D. Thesis, University College London (2012) 